\title{
Bright and dark vector rogue waves
}

\author{
Sergey V. Sergeyev ${ }^{1}$, Chengbo Mou ${ }^{1,2^{*}}$, Stanislav Kolpakov ${ }^{1}$, Vladimir Kalashnikov ${ }^{1}$ \\ ${ }^{1}$ Aston Institute of Photonic Technologies, Aston University, Aston Triangle, B4 7ET, Birmingham, UK \\ ${ }^{2}$ The Key Laboratory of Specialty Fiber Optics and Optical Access Network, Shanghai University, 200072, \\ Shanghai, China \\ ${ }^{*}$ Tel: +44 (0)1212043504, e-mail: mouc1@shu.edu.cn
}

\begin{abstract}
For an Erbium-doped mode locked fibre laser, we demonstrate experimentally a new type of vector rogue waves (RWs) emergence of which is caused by the coherent coupling of the orthogonal states of polarisation (SOPs). Unlike weak interaction between neighbouring dissipative solitons for the soliton rain, this creates a new type of the energy landscape where the interaction of the orthogonal SOPs leads to polarisation trapping or escapes from the trapping triggered by polarisation instabilities and so results in the pulse dynamics satisfying criteria of the "dark" and "bright" RWs. The obtained results, apart from the fundamental interest, can provide a base for development of the rogue waves mitigation techniques in the context of the applications in photonics and beyond.
\end{abstract}

Keywords: mode locked lasers, rogue waves, polarisation

\section{INTRODUCTION}

The femtosecond/picosecond pulsewidth and $\mathrm{MHz}$ repetition rate of mode locked fibre lasers (MLFLs) provide an opportunity to observe more data on rogue waves in the form of the chaotic bunches of pulses (soliton rain [1]-[5]) in the short time (compared to the time scale of RWs in other systems, such as in the ocean [6]-[8]) and under controlled conditions. This makes such lasers perfect test bed systems to study RWs in the context of origin and mitigation with further results application in numerous disciplines - social sciences, natural sciences and technology \& engineering [1-5]. In MLFLs, adjacent pulses interact through their tails overlapping or through the dispersive waves. Such interaction can be controlled by the pump power modulation or/and by injection of a weak seeding signal and noise that is similar to the fluctuation induced escape (FIE) phenomena in an energy landscape (EL) (set of equilibrium minima and saddle points, i.e. unstable maxima in the potential) of the multistate or excitable systems [9]-[10]. Based on the weak interaction model (WIM), such scenario of RWs emergence has been recently outlined theoretically based on the nonlinear Schrödinger (NLS) equation [11]. We support the concept in this paper by providing an experimental evidence of a new type of the energy landscape created by the interaction of the orthogonal states of polarisation (SOPs) and leading to the polarisation trapping or escape from the trapping which is triggered by polarisation instabilities [12]-[13]. We demonstrate that tuning the in-cavity and the pump polarisation controllers, i.e. cavity birefringence and the ellipticity of the pump wave, enable transitions between two orthogonal states of polarisation which leads to output power distribution satisfying the rogue wave's criteria.

\section{RESULTS AND DISCUSSION}

The laser (Fig. 1) consists of $1.1 \mathrm{~m}$ long erbium doped fibre (EDF) with a nominal absorption ratio of $80 \mathrm{~dB} / \mathrm{m}$ at $1530 \mathrm{~nm}$. The group velocity dispersion of the EDF is $\sim+59 \mathrm{ps}^{2} / \mathrm{km}$. A fibre pigtailed optical isolator (OISO) is used to provide a unidirectional operation of the laser. A $975 \mathrm{~nm}$ laser diode (LD) with the output power of 67 $\mathrm{mW}$ is employed to pump the laser via a 980/1550 wavelength division multiplexer (WDM). This power is 10 times less than the pump power used by many authors for the experimental and theoretical study of the soliton rain [1]-[5]. A standard 70:30 fused coupler is used to extract 30\% of the laser light out of the cavity. The rest of the laser cavity contains $1.22 \mathrm{~m}$ OFS980 fibre and 4.4m SMF 28 fibre. The CNT mode-locker is a piece of the carbon nanotube polymer composite film sandwiched between two standard fibre connectors. The index matching gel was used to minimise the insertion losses. The total cavity GVD is $\sim-0.04 \mathrm{ps}^{2} / \mathrm{nm}$. Two polarisation controllers (POC1 and POC2) were used to adjust the SOP of the pump wave and the in-cavity birefringence. The polarimeter (Thorlabs IPM5300) was mounted on a chassis (Thorlabs TXP - 5016) and tuned to have a $1 \mu \mathrm{s}$ resolution and a total temporal length of the sample of $1 \mathrm{~ms}$ (1024 points per measurement). This polarimeter was used to measure the normalised Stokes parameters $s_{1}, s_{2}, s_{3}$, the degree of polarisation (DOP) and the power of the signal. The laser signal was measured using a $U D P-15-I R-2 F C$ detector with a bandwidth of $17 \mathrm{GHz}$; the electric signal from the detector was recorded with a Tektronix DPO7254 - $2.5 \mathrm{GHz}$ oscilloscope. The POC1 and POC2 have been adjusted to find conditions for RWs emergence. The optical spectrum was measured with the optical spectrum analyser (ANDO AQ6317B) and the pulse width - with the help of the auto-correlator (Pulsecheck). The results of measurements are shown in Figs.2 and 3. The oscillogram comprises 20 slices of the pulse dynamics with $20 \mathrm{~K}$ points in each slice, autocorrelation trace consists of 8 slices, and polarimeter trace includes 20 slices. 
As follows from Fig. 2 (a), multi-pulse dynamics is stable during each slice (16 roundtrips) and is changing from slice to slice. Detailed auto-correlation analysis (Fig. 2 (b)) and the shape of optical spectrum (Fig. 2 (c)) reveal soliton rain similar to the observed in literature [1-5]. The main difference is in low pump power of 67 $\mathrm{mW}$ used here vs $800 \mathrm{~mW}$ used by the other authors [1-5]. The pulse width is about $700 \mathrm{fs}$ and the distance between the main pulse and satellites fluctuates between 1 to $7 \mathrm{ps}$. The presence of the pedestal in autocorrelation trace and the absence of the pronounced spectral fringes indicate the presence of loosely bound solitons moving in the range of $1 \mathrm{ps}-10 \mathrm{~ns}$ delays with respect to the main pulse. Probability distribution histogram and RW criteria (more than eight standard deviations from the mean value, i.e. mean $(I)+8 \sigma(I)$ where $I$ is an output power signal) are shown in Fig. 2 (d). As follows from this figure, our case corresponds to the presence of the rogue waves.

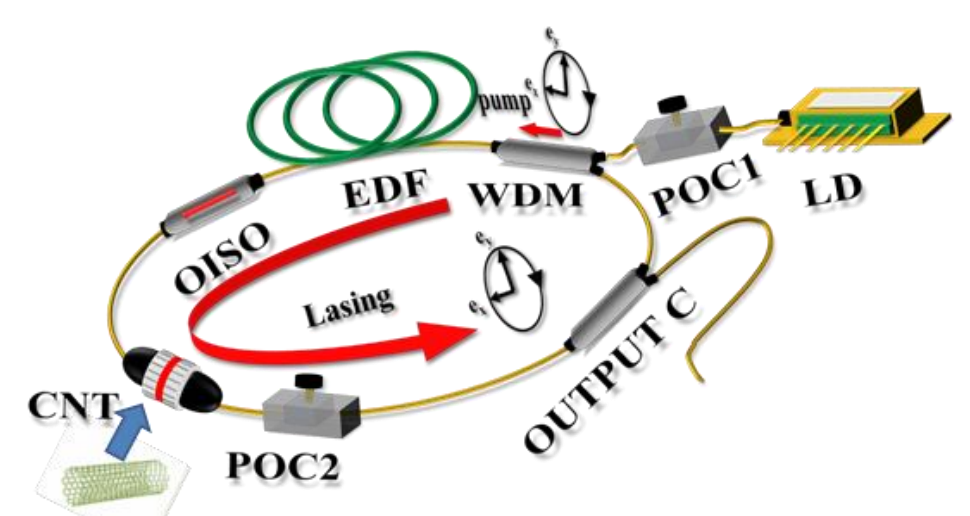

Figure 1 Experimental set-up.

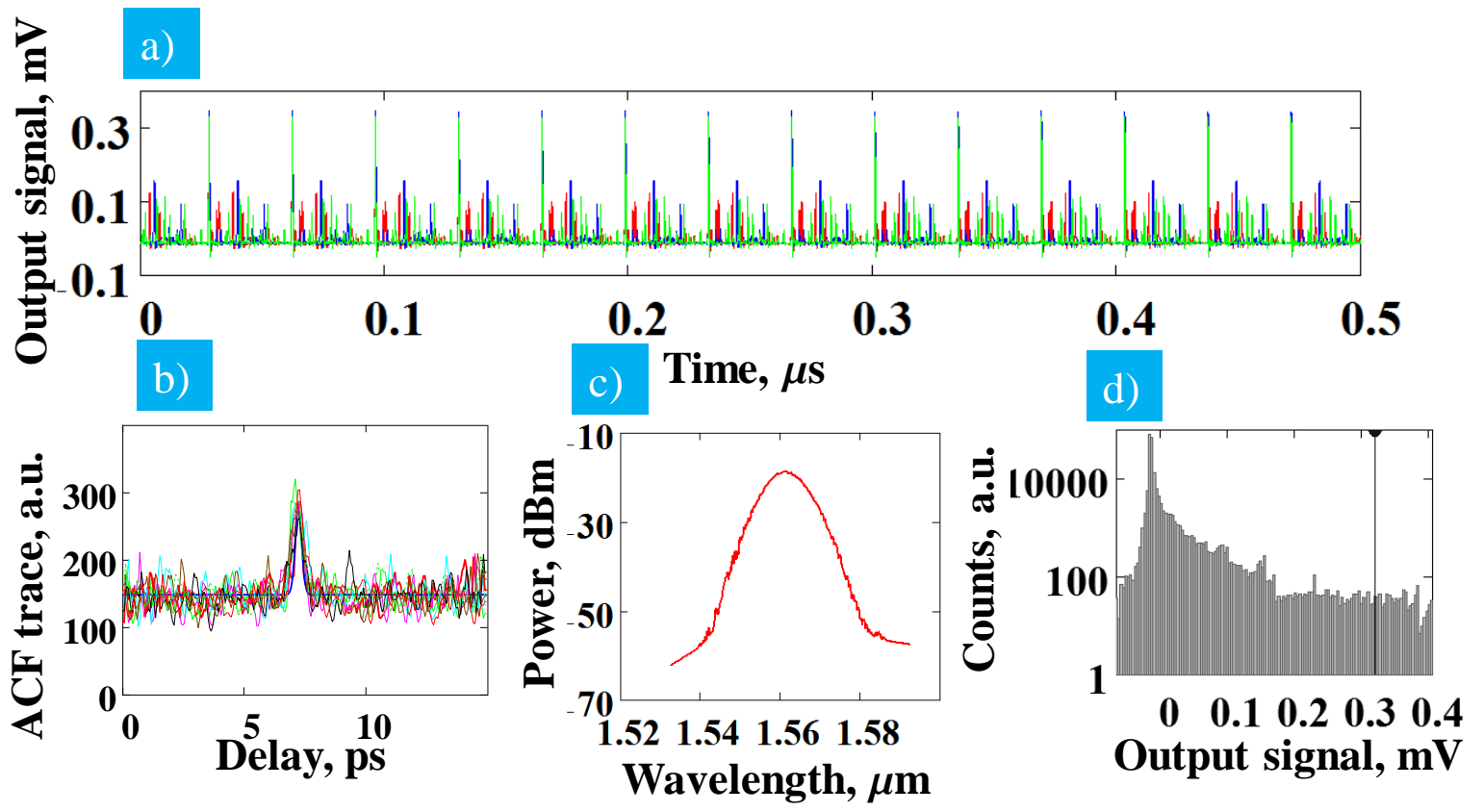

Fig.2 a) Oscillogram (2.5 GHz resolution): 3 different traces are shown in different colours, $b$ ) autocorrelation trace: different slices are shown in different colours, $c$ ) optical spectrum, d) probability distribution histogram: $R W$ criteria (mean $(I)+8 \sigma(I))$ is shown as the vertical line.

To get insight ino the mechanism of the RW emergence we used the polarimeter with $1 \mu$ s resolution and interval of $1 \mathrm{~ms}$ (aprox $30-30000$ round trips range) to measure the normalized Stokes parameters $s_{1}, s_{2}, s_{3}$ which are related to the output powers of two linearly cross-polarized SOPs $I_{x}$ and $I_{y}$, and phase difference between them $\Delta \phi$ as follows:

$$
S_{0} \equiv I=I_{x}+I_{y}, S_{1}=I_{x}-I_{y}, S_{2}=2 \sqrt{I_{x} I_{y}} \cos \Delta \phi, S_{3}=2 \sqrt{I_{x} I_{y}} \sin \Delta \phi, S_{i}=\frac{S_{i}}{\sqrt{S_{1}^{2}+S_{2}^{2}+S_{3}^{2}}},(i=1,2,3) .
$$


Results are shown in Fig. 3 (a-c). As follows from Fig. 3 (a), the anomalous spikes in the output power corresponds to the phase difference jumps in $\pi$, i.e. transitions between orthogonally polarised SOPs as follows from Fig. 3 (b). The probability distribution histogram is shown in Fig. 3 (c) along with RW criteria (red and blue lines) indicate the presence of so-called "bright" (spikes in Fig.3 (a)) and "dark" (dips in Fig. 3 (a)) RWs (Fig3 (c)). As follows from Figs. 2 and 3, slow evolution of the soliton rain is accompanied by the random polarisation switching between orthogonal SOPs. As a result, averaging over the 30 roundtrips provide almost constant output power (Fig.3 (a)) for different structures of the soliton rain (Fig. 2 (a)) whereas it is likely that spikes and dips appear when the type of the structure changes (Fig. 3 (a)) as result of the abrupt switching. The mechanism of such vector rogue waves emergence can be caused by the chaotic phase drift considered by Ansmann and co-workers for the system of two coupled oscillators [14].

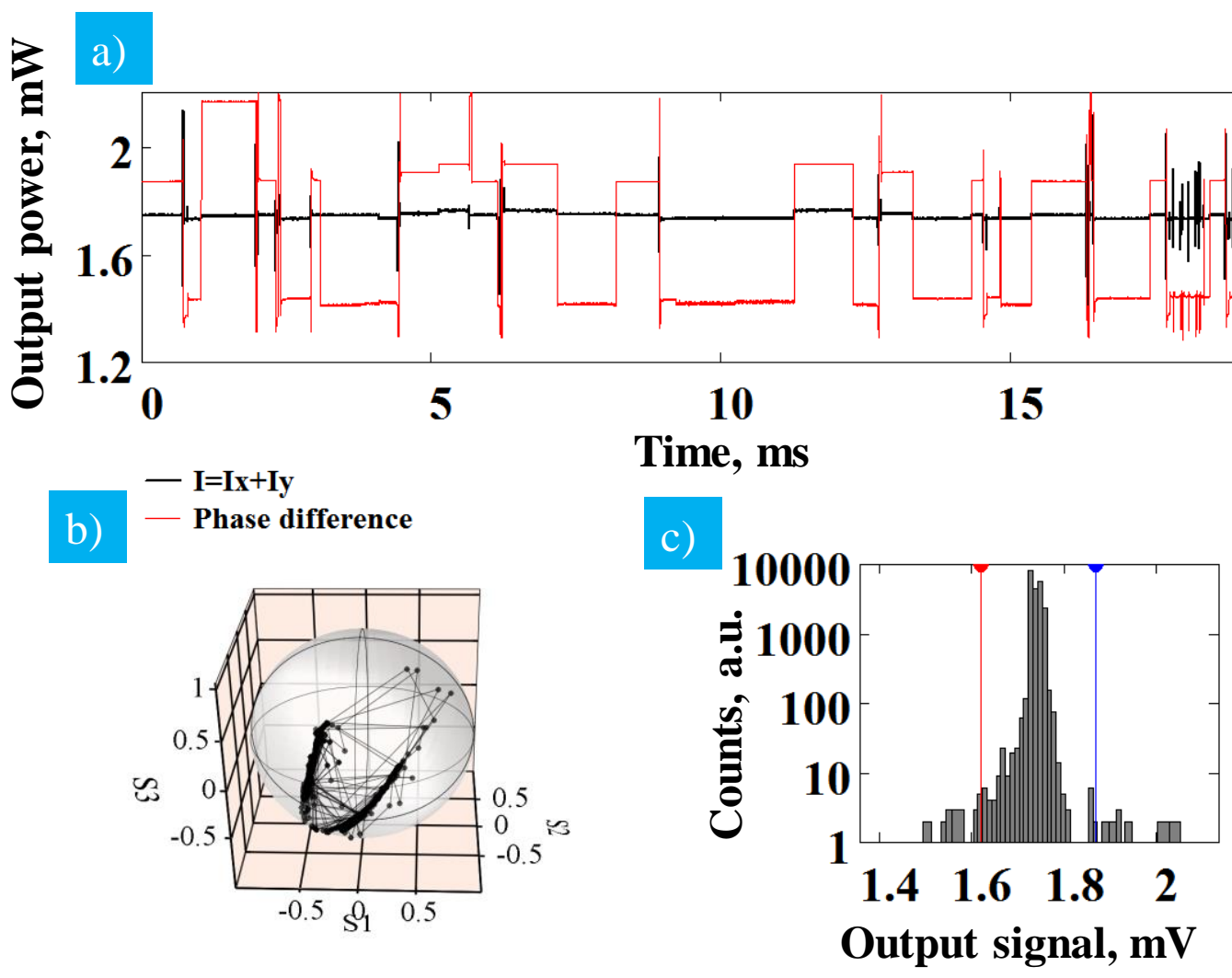

Fig. 3 Polarization measurements ( $1 \mu$ s resolution, i.e. averaging over approximately 33 round trips): a) The output power and the phase difference vs time, $b$ ) trajectories in normalised Poincaré sphere, and c) probability distribution of the output power (red and blue lines are the "dark" and "bright" RWs criteria).

Models of networks of coupled oscillators are widely used to study different synchronisation scenarios from the phase locking to the phase drift in the context of applications from biomedicine to the laser physics [15]. For the phase couple oscillators, evolution can be considered based on Kuramoto equations:

$$
\frac{d \varphi_{i}}{d t}=\Omega_{i}+\frac{K}{N} \sum_{i=1, i \neq j}^{N} G\left(\varphi_{i}-\varphi_{j}\right)
$$

Here $\phi_{i}$ and $\Omega_{i}$ are the phase and the frequency of the oscillator, $K$ is the coupling strength and $G$ is the coupling function [15]. In the simplest case of two coupled oscillators with $G=\sin \left(\phi_{i^{-}} \phi_{j}\right)$, Eq. (2) is called Adler equation [15]. For a comprehensive study of the synchronisation in lasers leading to complex dynamics along with conditions for RWs emergence, the amplitude dynamics along with dynamics of the population inversion have to be included into consideration that results in a new class of coupled system, i.e. coupled oscillators with the dynamic coupling strength [15]. With accounting for the SOP, orthogonally polarised SOPs can be treated as coupled oscillators as well $[12,13]$. This provided a very good test bed for study synchronisation regimes of the orthogonal SOPs in a mode-locked fibre laser with a saturable absorber as a function of the laser parameters (power and ellipticity of the pump wave, and in-cavity birefringence) tunable by the pump laser current driver, polarisation controller for pump wave and in-cavity polarisation controller [5-8]. The result of the laser 
parameter tuning shown in Fig. 3 (a) leads to chaotic phase jumps of the phase difference between the orthogonal SOPs which coincide with the anomalous spikes and dips in the output power. Thus, the observed in Fig. 3 rare events taking the form of the "dark" and "bright" vector RWs along with quite long time intervals with the constant output power can be explained in terms of the spontaneous desynchronization of the cross polarised SOPs. The desynchronization can be caused by the decreased coupling between SOPs allowing the polarisation instabilities-driven chaotic transitions.

\section{CONCLUSIONS}

We have demonstrated experimentally a new class of the vector rogue waves taking the form of the rare anomalous switching between different types of the soliton rain. This type of switching has a time scale of tenths-hundreds roundtrips and can take a form of the "dark" and "bright" vector rogue waves. We show that tuning in-cavity and the pump polarisation controllers, i.e. the cavity birefringence and the ellipticity of the pump wave, enable, in terms of the theory of the coupled oscillators, changing the coupling strength between two orthogonal SOPs and detuning between their oscillation frequencies that is finally result in polarisation trapping or escape from the trapping triggered by polarisation instabilities [12]-[13]. The revealed conditions for emergence of new class of the rogue waves can potentially find applications for controlling synchronisation scenarios along with rogue wave emergence in the ensemble of coupled oscillators that mimic a wide range of the coupled systems such as multimode and coupled lasers, electrical power grids, communication networks, human brain, and financial markets.

\section{ACKNOWLEDGEMENTS}

This work was financed by the Leverhulme Trust (Grant ref: RPG - 2014 - 304) and the FP7-PEOPLE-2012IAPP (project GRIFFON, No. 324391).

\section{REFERENCES}

[1] C. Lecaplain, Ph. Grelu, J.M. Soto-Crespo, and N. Akhmediev: Dissipative Rogue Waves Generated by Chaotic Pulse Bunching in a Mode-Locked Laser, Phys. Rev. Lett., vol. 108 , p. 233901 , Jun. 2012.

[2] C. Lecaplain, Ph. Grelu, J.M. Soto-Crespo, and N. Akhmediev: Dissipative rogue wave generation in multiple-pulsing mode-locked fiber laser, J. Optics, vol. 15, p. 064005, Jun. 2013.

[3] A. Zavialov, O. Egorov, R. Iliev, and F. Lederer: Rogue waves in mode-locked fiber lasers, Phys. Rev. A vol. 85, pp. 013828, Jan. 2012.

[4] A. Niang, et.al.: Rains of solitons in a figure-of-eight passively mode-locked fibre laser, Appl. Phys. B, vol. 116, pp. 771-775, Jan. 2014.

[5] F. Sanchez, et al.: Manipulating dissipative soliton ensembles in passively mode-locked fiber lasers. $O p t$. Fiber Techn., vol. 20, pp.562-574, Dec. 2014

[6] C. Kharif, E. Pelinovsky, A. and Slunyaev: Rogue Waves in the Ocean, Heidelberg, Springer, 2009.

[7] A. Slunyaev et al.: Super-rogue waves in simulations based on weakly nonlinear and fully nonlinear hydrodynamic equations, Phys. Rev. E, vol. 8, p. 012909, Jul. 2013.

[8] A. R. Osborne: Nonlinear Ocean Waves and the Inverse Scattering Transform, Academic Press, 2010.

[9] B. Lindner, J. Garsia-Ojalvo, A. Neiman, L. Schimansky-Greif: Effects of noise in excitable systems, Phys. Reports, vol. 392, pp.321-424, Mar. 2004.

[10] L. Gammaitoni, P. Hänggi, P. Jung, and F. Marchesoni: Stochastic resonance, Rev. Mod. Phys., vol. 70, pp. 223-287, Jan. 1998.

[11] A. Armaroli, C. Conti, and F. Biancalana: Rogue solitons in optical fibers: a dynamical process in a complex energy landscape, Optica, vol. 2, pp. 497-503, May 2015.

[12] S. V. Sergeyev et al.: Spiral attractors created by vector soliton, Light: Science \& Appl., vol. 3, p. e131, Jan. 2014.

[13] S. V. Sergeyev: Fast and slowly evolving vector solitons in mode locked fibre laser, Phil. Trans. R. Soc. A vol. 372, p. 20140006, Oct. 2014.

[14] G. Ansmann, R. Karnatak, K. Lehnertz, and U. Feudel: Extreme events in excitable systems and mechanisms of their generation, Phys. Rev. E, vol.88, p. 052911, Nov. 2013.

[15] A. Arenas et al.: Synchronization in complex networks, Phys. Rep., vol.469, pp.93-153, Sep.2008. 